\title{
APRENDIZAGEM SOCIAL E DESENVOLVIMENTO SUSTENTÁVEL: UM ESTUDO BIBLIOMÉTRICO NA WEB OF SCIENCE DA ÚLTIMA DÉCADA
}

\author{
SOCIAL LEARNING AND SUSTAINABLE DEVELOPMENT: \\ A BIBLIOMETRIC STUDY ON THE WEB OF SCIENCE OF THE LAST DECADE
}

\author{
RODRIGO REIS FAVARIN \\ Universidade Federal de Santa Maria (UFSM) \\ Doutorando no Programa de Pós Graduação em Administração (UFSM) \\ Orcid: https://orcid.org/0000-0001-8591-5733 \\ E-mail: rodrigo.favarin@hotmail.com
}

Rua Olavo Bilac, 418. Apto 301. Bairro: Nossa Senhora de Fátima- CEP:97015-440 Santa Maria/RS

\author{
NATHÁLIA RIGUI TRINDADE \\ Universidade Federal de Santa Maria (UFSM) \\ Doutoranda no Programa de Pós Graduação em Administração (UFSM) \\ Orcid: https://orcid.org/0000-0003-1361-6501 \\ E-mail: nathaliariguitrindade@gmail.com \\ LUCAS VEIGA ÁVILA \\ Professor na Universidade Federal de Santa Maria (UFSM) \\ Titulação: Doutor em Administração \\ Orcid: https://orcid.org/0000-0003-1502-258X \\ E-mail: admlucasveiga@gmail.com \\ MARCELO TREVISAN \\ Professor na Universidade Federal de Santa Maria (UFSM) \\ Titulação: Doutor em Administração \\ Orcid: https://orcid.org/0000-0002-2403-7970 \\ E-mail: marcelotrevisan@smail.ufsm.br
}

Submissão: 22/05/2019. Revisão: 07/07/2020. Aceite: 23/07/2020. Publicação: 03/08/2020.

DOI: http://dx.doi.org/10.22277/rgo.v13i3.4906

\begin{abstract}
RESUMO
Este estudo buscou analisar as características das publicações sobre social learning (aprendizagem social) e sustainable development (desenvolvimento sustentável) na base de dados Web of Science, no período de 2009 a 2018 e, ainda, identificar quais os tópicos mais relevantes relacionados à aprendizagem social e ao desenvolvimento sustentável que estão sendo investigados (hot topics). 0 trabalho descritivo e quantitativo, de natureza bibliométrica, busca levantar as características da produção acadêmica. Como principais resultados, das 2.024 publicações analisadas no período, constatou-se um crescimento sobre a produção científica ao longo da última década, em especial nas seguintes áreas temáticas: Pesquisa educacional, Ciências ambientais e ecologia, Estudos ambientais, Tecnologia e ciências sustentáveis verdes e Gestão. Evidenciou-se como tópicos quentes as combinações do tópico Aprendizagem social e Desenvolvimento sustentável com Gestão, Estudos de desenvolvimento, Estudos ambientais e Ciências ambientais. Por fim, verifica-se que a aprendizagem social contribui com grande avanço para área do desenvolvimento sustentável, em especial para a educação e ciência ambiental, com novas formas de aprendizagem para a sustentabilidade.
\end{abstract}

Palavras-chave: Aprendizagem Social. Desenvolvimento Sustentável. Bibliometria. 
Aprendizagem social e desenvolvimento sustentável: um estudo bibliométrico no Web of Science da última década

\begin{abstract}
This study sought to analyze the characteristics of publications on social learning (sustainable learning) and sustainable development (sustainable development) in the Web of Science database, from 2009 to 2018, and also to identify which are the most relevant topics related to social learning and sustainable development that are being investigated (hot topics). Descriptive and quantitative work, of a bibliometric nature, seeks to raise the characteristics of academic production. As the main results of the 2,024 publications analyzed in the period, there was an increase in scientific production over the last decade, especially in the following thematic areas: Educational research, Environmental sciences and ecology, Environmental studies, Technology and green sustainable sciences and Management. The hot topics were the combinations of the topic Social Learning and Sustainable Development with Management, Development Studies, Environmental Studies and Environmental Sciences. Finally, it appears that social learning contributes with great progress to the area of sustainable development, especially for education and environmental science, with new forms of learning for sustainability.
\end{abstract}

Keywords: Social learning. Sustainable development. Bibliometrics.

\title{
1 INTRODUÇÃO
}

À medida que a crise socioecológica se expande pelo mundo, impactando nos sistemas socioculturais e ecológicos, a busca pelo desenvolvimento sustentável (DS) se torna mais urgente. $\mathrm{O}$ termo desenvolvimento sustentável tem sido comumente adotado para designar um modelo ideal de desenvolvimento que seja economicamente viável, ecologicamente prudente e socialmente justo (CAVAGNARO; CURIEL, 2012).

Com o aumento da consciência em relação às ações que devem ser tomadas em prol do DS, três atores possuem um papel especialmente importante: o Estado, intervindo e contribuindo com políticas que favoreçam este desenvolvimento; a sociedade por meio da governança adaptativa (aprendizado, inovação, compartilhamento de informações) e; as organizações gerando desenvolvimento econômico que dialogue com a comunidade e, também, atuando com responsabilidade socioambiental (XAVIER et al., 2013). A partir disso, Cars e West (2014) defendem que o DS demanda mudanças de valores e atitudes da população, sendo a educação a peça chave para transformações. Isto pois, uma educação para o desenvolvimento sustentável (EDS) busca promover uma série de competências como o pensamento crítico, a capacidade de projetar cenários futuros e de tomar decisões de maneira colaborativa.

Para tanto, diferentes abordagens educacionais podem ser utilizadas na promoção da EDS. Uma delas, a Teoria da aprendizagem social, tem ganhado importância na busca por soluções educacionais que promovam o DS (CAPRA, 2007; GLASSER, 2007; HARM ONICOP, 2003; JACOBI, 2010; KEEN; BROWN; DYBALL, 2005; KILVINGTON, 2007; KILVINGTON, 2010; WALS; VAN DER LEIJ, 2007). Estes autores podem ser considerados os precursores dos estudos da aprendizagem social para sustentabilidade, por meio das suas investigações relacionadas à gestão de recursos naturais e gestão ambiental. Posteriormente, outro grupo de pesquisadores expandiu o escopo dos estudos, direcionando a análise da aprendizagem social para ambientes que não se relacionavam apenas com recursos naturais (AMARAL; BRUNSTEIN, 2017; D'ANGELO; BRUNSTEIN, 2014).

Nesse sentido, Didham, Ofei-Manu e Nagareo (2017) defendem a aprendizagem social como uma estrutura capaz de orientar e ampliar a EDS em práticas coletivas e sociais e 
destacam que nas últimas décadas tem havido um crescimento expressivo da aplicação dessa abordagem teórica envolvendo diferentes questões. Rodela, Cundill e Wals (2012) destacam que embora o corpo de literatura sobre aprendizagem social tenha apresentado rápido crescimento, ainda não há uma clareza conceitual, o que torna a literatura, por muitas vezes, vaga sobre o conceito, aplicação e eficácia da aprendizagem social. É nesse contexto que questiona-se: quais as principais características do campo teórico que aborda a relação entre aprendizagem social e desenvolvimento sustentável nos últimos anos?

Uma vez que, é importante compreender a organização do estado da arte de uma área de estudo para a caracterização da produção intelectual daquele campo teórico (FAVORETO; AMÂNCIO-VIEIRA; SHIMADA, 2014), esse artigo busca levantar dados a respeito do corpo de publicações que abordam esta relação entre os temas (aprendizagem social e desenvolvimento sustentável). Assim, define-se o objetivo da presente pesquisa como analisar as características das publicações sobre social learning (aprendizagem social) e sustainable development (desenvolvimento sustentável) na base de dados Web of Science, no período de 2009 a 2018 e, ainda, identificar quais os tópicos mais relevantes relacionados à aprendizagem social e ao desenvolvimento sustentável que estão sendo investigados (hot topics). Para isso, realizou-se uma pesquisa bibliométrica, a qual possibilita a investigação de um número expressivo de informações da produção científica de um dado campo teórico.

A partir do desenvolvimento do estudo, espera-se contribuir na compreensão de como a aprendizagem social tem sido trabalhada nas pesquisas sobre DS, sintetizando características das produções científicas e elucidando a gama de conhecimentos que já foram investigados nesse campo do saber. Dessa forma, busca-se promover avanços na construção de novos conhecimentos sobre a aprendizagem social e desenvolvimento sustentável, fornecendo insights para pesquisadores, novas possibilidades de investigações e temáticas de estudo. Além disso, indicações de oportunidades de parcerias de pesquisa, também são possíveis, uma vez que busca identificar instituições, países, agências financiadoras e autores que se destacam nas publicações sobre o assunto. Em suma, os resultados poderão servir como fonte de inspiração para pesquisadores e profissionais interessados em utilizar a aprendizagem social no ensino e promoção do DS.

Para tanto, o presente artigo está estruturado em seis seções, considerando esta introdução a primeira delas. Na segunda seção, apresenta-se o referencial teórico que aborda as temáticas do desenvolvimento sustentável e da aprendizagem social. Já a terceira seção apresenta os procedimentos metodológicos utilizados para o desenvolvimento da pesquisa. Na sequência, a quarta e quinta seções apresentam, respectivamente, a análise dos resultados e as considerações finais. Por fim, encerra-se o estudo com as referências utilizadas.

\section{REFERENCIAL TEÓRICO}

\subsection{DESENVOLVIMENTO SUSTENTÁVEL}

O fato de que o planeta enfrenta dificuldades para suprir a alta demanda por seus recursos naturais já se tornou algo estabelecido na sociedade. O início das discussões e debates acerca de um desenvolvimento que levasse o meio ambiente em consideração pode ser atribuído ao conceito de ecodesenvolvimento, que teve origem ainda na década de 70 (ROMEIRO, 2012).

Porém, na década de 90, a Comissão Mundial sobre Meio Ambiente e Desenvolvimento (CMMAD) lançou o relatório de Brundtland, cujo título é atribuído a Gro Brundtland, sua 
Aprendizagem social e desenvolvimento sustentável: um estudo bibliométrico no Web of Science da última década

presidente e primeira-ministra da Noruega. O documento apresentou o termo desenvolvimento sustentável (DS), conceituando-o como aquele que "satisfaz as necessidades do presente sem comprometer as habilidades das futuras gerações de satisfazerem as suas necessidades" (COMISSÃO MUNDIAL SOBRE MEIO AMBIENTE E DESENVOLVIMENTO, 1991, p. 44). Desta forma, o desenvolvimento sustentável foi colocado no mapa internacional de discussões e pesquisas.

Apesar de vaga, a definição representa um compromisso político entre sustentabilidade ambiental e crescimento, adequada para as delegações das Nações Unidas (CASTRO, 2004). Corroborando com o tema, Hopwood, Mellor e O'Brien (2005) afirmam que o conceito do DS resulta da crescente conscientização dos elos globais entre questões socioeconômicas, problemas ambientais e preocupações com um futuro saudável para a humanidade. Já Seghezzo (2009) aponta quatro grandes limitações do conceito da CMMAD: a) é essencialmente antropocêntrico, no qual o bem-estar humano é a principal razão para a proteção do capital natural; b) a importância da economia é superestimada; c) espaço e tempo são negligenciados, pois os indicadores normalmente se referem a aspectos econômicos, sociais e ambientais e; d) o ser humano não pode ser equiparado apenas as suas necessidades.

Com o objetivo de facilitar o entendimento das questões ambientais e socioeconômicas no meio empresarial, John Elkington desenvolveu o conceito do tripé da sustentabilidade, que se refere aos três pilares do desenvolvimento sustentável: a) o pilar econômico, responsável pela produção de riqueza por meio da comercialização de bens e serviços; b) o pilar social, que almeja oportunizar a igualdade entre os grupos sociais envolvidos por meio do cumprimento e preocupação das responsabilidades e direitos dos indivíduos; c) pilar ambiental, remete à conservação e manutenção dos recursos naturais disponíveis (ELKINGTON, 1999). Outro modelo mundialmente conhecido, foi desenvolvido por Ignacy Sachs, no qual o autor estabelece um total de oito dimensões para o desenvolvimento sustentável, a saber: social, cultural, ecológico, ambiental, territorial, econômico, político nacional e político internacional (SACHS, 2004).

Não obstante aos diversos modelos e conceitos criados, existem críticas acerca da temática do DS (CASTRO, 2004; FOLADORI, 2001; HOPWOOD; MELLOR; O’BRIEN, 2005; SEGHEZZO, 2009). Para Castro (2004), a natureza é vista como um recurso que deve ser utilizado com eficiência, e não como algo que deve ser respeitado, sob o paradigma do DS. Ainda para o autor, as organizações buscam exercer controle social sobre o capital, utilizando o conceito do DS, e desta forma, contemplam o meio ambiente apenas como uma preocupação subsidiária ao promover o crescimento econômico, seu principal objetivo.

Outra questão abordada por parte dos pesquisadores relaciona os problemas socioambientais com o sistema econômico vigente em grande parte do planeta: o capitalismo (CASTRO, 2004; FOLADORI, 2001). Castro (2004) afirma que o consumismo e a acumulação de capital impulsionam a economia capitalista, de maneira a sustentarem o desenvolvimento, ao invés de desenvolverem a sustentabilidade no sentido ecológico. Foladori (2001) vai além, evidenciando que a concorrência mercantil, implica em determinados comportamentos em relação ao meio ambiente, de maneira a extrapolar os limites físicos naturais. Ainda para o autor, alia-se ao problema questões como superpopulação, pobreza, depredação, poluição, entre outros.

Hopwood, Mellor e O’Brien (2005) observam a falta de uma filosofia única e unificada para a promoção do DS, pois muitas vezes, há equívocos no emprego de palavras, o que gera divergência de pontos de vistas sobre os objetivos, rotas e métodos de avançar no desenvolvimento do trabalho. 
Com o intuito de reduzir incertezas e centralizar as ações em prol do DS, as Nações Unidas criaram os Objetivos do Desenvolvimento Sustentável (ODS). Eles se referem a 17 objetivos, divididos em 169 metas que pretendem "estimular a ação nos próximos 15 anos em áreas de importância crítica para a humanidade e o planeta" (ONU, 2015, p. 3). Griggs et al. (2014) reconhecem que os ODS, em sua totalidade, integram as dimensões sociais, econômicas e ambientais que sustentam o conceito de DS.

Para Bebbington e Unerman (2017), a maneira como os ODS estão estruturados deixa evidente que somente a ação governamental não é suficiente e que será necessário o envolvimento dos setores públicos e privados, sociedade civil e cidadãos individuais, para que as metas sejam atingidas. Da mesma forma, Castro Filho (2018) afirma que a agenda se origina do trabalho conjunto de governos e cidadãos de todo o mundo para criar um modelo global que busca acabar com a pobreza, promover a prosperidade e o bem-estar de todos, proteger o ambiente e combater as alterações climáticas.

E, é neste sentido que Bebbington e Unerman (2017) afirmam que os ODS revelam alguns desafios à comunidade acadêmica, principalmente no que tange a três aspectos: i) retomar a justiça econômica como foco; ii) colocar a responsabilidade ecológica como temática central e; iii) renovar o foco nas responsabilidades do ensino superior nesse processo de alcance das metas.

Pode-se dizer, então, que é importante que o processo de construção de conhecimento e aprendizagem sejam temas centrais no movimento em busca dos ODS, aonde a ação conjunta de todos os sujeitos, com suas especificidades e know-how, possa gerar resultados que superem as críticas recebidas quanto a exequibilidade dos objetivos e compromissos ideológicos inerentes aos mesmos (BEBBINGTON; UNERMAN, 2017). Com isso, a partir dos principais conceitos e discussões sobre o desenvolvimento sustentável, a próxima seção deste estudo abordará a aprendizagem social, que recentemente tem sido evidenciada como de importância na promoção do desenvolvimento sustentável (GLASSER, 2007; JACOBI, 2010; KILVINGTON, 2010).

\subsection{APRENDIZAGEM SOCIAL}

A Teoria da Aprendizagem Social apresenta-se com uma importante abordagem teórica para identificar quais são e como ocorrem os processos de aprendizagem entre os indivíduos e os diversos níveis sociais. O teórico Albert Bandura, começou a desenvolver a Teoria da Aprendizagem social no ano de 1962. Em 1977, ele aprofundou os seus estudos com o lançamento da Teoria da Auto-eficácia e, em 1986 a denominou Teoria Social Cognitiva, quando lançou o livro Social Foundations of Thought and Action (Fundações Sociais do Pensamento e Ação).

Originalmente, o conceito da aprendizagem social se refere à aprendizagem que os indivíduos adquirem em contextos sociais por meio da observação e imitação dos outros (BANDURA, 1977). O autor avalia que a concepção original da teoria se apoia em um processo individual, trazendo à tona que a maior parte do comportamento humano é aprendizagem pela observação por meio da modelagem. Segundo o autor, pela observação dos outros, uma pessoa forma uma ideia de como novos comportamentos são executados e, em ocasiões posteriores, esta informação codificada serve como um guia para a ação.

Para D'Angelo e Brunstein (2014), com a evolução dos seus estudos, Bandura expandiu as suas concepções e afirmava que o comportamento humano é explicado por meio das influências das estruturas comportamentais, cognitivas e ambientais. Ou seja, o lócus da aprendizagem são as interações sociais entre indivíduos e ambiente. Para Bandura (1977), 
Aprendizagem social e desenvolvimento sustentável: um estudo bibliométrico no Web of Science da última década

uma modelagem comportamental bem-sucedida é composta por quatro etapas, que estão representadas no Quadro 1.

Quadro 1 - As quatro etapas de uma modelagem comportamental bem-sucedida

\begin{tabular}{|c|l|}
\hline Etapa & \multicolumn{1}{c|}{ Processo } \\
\hline Atenção & $\begin{array}{l}\text { Para aprender, é necessário prestar atenção; } \\
\text { Qualquer distração irá afetar negativamente na aprendizagem por observação; } \\
\text { Se o modelo é interessante ou há um aspecto novo da situação, é muito mais provável a total } \\
\text { dedicação à aprendizagem. }\end{array}$ \\
\hline \multirow{5}{*}{ Retenção } & $\begin{array}{l}\text { A capacidade de armazenar informações também é uma parte importante do processo de } \\
\text { aprendizagem; } \\
\text { A retenção pode ser afetada por uma série de fatores, mas a capacidade de extrair } \\
\text { informações mais tarde e atuar sobre ela é vital para a aprendizagem observacional. }\end{array}$ \\
\hline \multirow{2}{*}{ Meprodução } & $\begin{array}{l}\text { Uma vez que se prestou atenção ao modelo e manteve-se a informação, é hora de realmente } \\
\text { realizar o comportamento observado; } \\
\text { A prática adicional do comportamento aprendido leva a melhoria e progresso de habilidades. }\end{array}$ \\
$\begin{array}{l}\text { Finalmente, para que a aprendizagem observacional seja bem-sucedida, a motivação é } \\
\text { necessária para que se imite o comportamento modelado; } \\
\text { O reforço e a punição desempenham um papel importante na motivação. Embora a } \\
\text { experimentação desses motivadores possa ser altamente efetiva, pode-se observar outros } \\
\text { que experimentam algum tipo de reforço ou punição. Por exemplo, se um estudante vê outro } \\
\text { sendo recompensado, com nota extra por estar na sala de aula no horário certo, ele poderá } \\
\text { começar a aparecer também no horário correto. }\end{array}$ \\
\hline
\end{tabular}

Fonte: Adaptado de Bandura (1997).

Outros autores que também estudaram a aprendizagem a partir de contextos sociais são Kurt Lewin e John Dewey. De acordo com Godoi e Freitas (2008), Lewin realizou pesquisas relacionadas às dinâmicas de grupos, e afirma que a conduta dos indivíduos em grupo é determinada pela dinâmica dos fatos e dos valores, que se percebe em cada situação, corroborando o pensamento de Bandura (1997) de que a observação é uma importante fonte da aprendizagem. Estas conclusões demonstram a importância do contexto, ou seja, do ambiente social no comportamento dos indivíduos em grupos.

Nas palavras de Reed et al. (2010) a aprendizagem social pode ser definida como uma transformação de entendimento dentro de unidades sociais amplas ou comunidades de prática por meio de interações sociais entre atores dentro de redes sociais, indo além do nível individual. As comunidades de práticas podem ser entendidas como grupos de pessoas que compartilham uma preocupação ou paixão por algo que fazem e aprendem como fazê-lo melhor, pois interagem regularmente (WENGER; TRAYNER, 2015).

Torna-se importante salientar que além de ocorrer por meio de interações sociais e processos entre os atores de uma rede social, a aprendizagem social necessita da certificação de que a mudança ocorreu na compreensão dos indivíduos envolvidos no processo, seja via novas informações, crenças ou atitudes e a percepção de que a mudança vai além dos indivíduos e se espalha dentro de unidades sociais mais amplas ou comunidades de prática (REED et al., 2010).

D'Angelo e Brustein (2014, p. 36) discorrem ainda sobre a noção de campo social, termo criado por Lewin, como sendo um "espaço onde as pessoas, os objetos, as instituições, os grupos e os acontecimentos sociais são elementos das situações sociais, que mantêm entre si relações dinâmicas". Já Dewey, importante representante do pragmatismo, afirma que a aprendizagem deve ser entendida como uma "contínua reorganização e reconstrução da experiência" que ocorre "todo o tempo e em todas as situações em que as pessoas agem e interagem - refletem e pensam". Trata-se de uma experiência reflexiva originada em uma situação-problema que leva o indivíduo a parar e pensar (D'ANGELO; BRUSTEIN, 2014, p. 37).

RGO - Revista Gestão Organizacional, Chapecó, v. 13, n. 3, p. 184-203, set./dez. 2020. 
Portanto, a aprendizagem ocorre por meio da reorganização e reconstrução da sua experiência.

Para Amaral e Brunstein (2017), o indivíduo é identificado como entidade base da aprendizagem. O contexto social e as capacidades cognitivas individuais determinam a aprendizagem, diante disso a importância da interação recíproca entre indivíduo-contexto para mudanças. De outro modo, quando os indivíduos são incapazes de influenciar o contexto por meio do seu comportamento, todo o sentido de tradução de conhecimento em ação desaparece. Logo, se o contexto é incapaz de mudar o indivíduo, percebe-se a continuidade de práticas antigas.

Em essência, a aprendizagem social trata-se de reunir pessoas de diferentes origens, no qual o conjunto de perspectivas, conhecimentos e experiências produzidos desta maneira necessita alcançar uma busca criativa de respostas para perguntas nas quais soluções prontas não estão disponíveis (WALS; VAN DER HOEVEN; BLANKEN, 2009).

\section{MÉTODO}

\subsection{TIPO DO ESTUDO}

O presente estudo foi desenvolvido a partir de uma pesquisa bibliométrica (ROSTAING, 1997; SILVA, 2004; MACEDO; CASA NOVA; ALMEIDA, 2009) de cunho quantitativo (MALHOTRA, 2006; HAIR et al., 2005), objetivando ampliar o conhecimento referente às publicações relacionadas à aprendizagem social (social learning) e ao desenvolvimento sustentável (sustainable development), na base de dados Web of Science, no período de 2009 a 2018. O estudo buscou analisar as características das publicações e identificar quais tópicos relacionados a estes termos estão sendo estudados junto a setores e quais são mais relevantes.

Além da literatura sobre bibliometria citada, e que fornece suporte para o desenvolvimento da pesquisa, cabe salientar que existem outros estudos que se destacam na área da administração. Cita-se o estudo de Moretti e Campanário (2009), Avila et al. (2018), os quais realizam uma classificação e análise estatística de obras citadas nas referências bibliográficas ou citações utilizadas pelos autores, particularmente com o confronto destas informações com a Lei de Lotka (LL), que é um princípio que estuda sobre a produtividade dos autores de um determinado campo científico. Esta lei da produtividade parte da premissa de que, proporcionalmente, poucos autores publicam mais (e são mais citados) do que muitos autores, que publicam menos. Esta distribuição é verificada em campos avançados da ciência. Moretti e Campanário apud Alvarado (2002) salienta que esta lei tem a seguinte definição: para estabelecer a parte com que seres humanos de diferentes qualidades contribuem ao progresso da ciência, Lotka estabeleceu os fundamentos da lei do quadrado inverso, afirmando que o número de autores que fazem $n$ contribuições em um determinado campo científico é aproximadamente $1 / \mathrm{n}^{2}$ daqueles que fazem uma só contribuição. A proporção daqueles que fazem uma única contribuição é de mais ou menos $60 \%$.

\subsection{DEFINIÇÃO DA AMOSTRA}

Os dados para a realização desta pesquisa foram coletados por meio da base Web of Science do Institute for Scientific Information (ISI). Torna-se importante destacar que a Web of Science consiste em uma base multidisciplinar, que indexa somente periódicos com grande número de citações na web, oferecendo informações sobre o impacto e a visibilidade das 
Aprendizagem social e desenvolvimento sustentável: um estudo bibliométrico no Web of Science da última década

publicações científicas, abrangendo aproximadamente 12.000 periódicos (COORDENAÇÃO DE APERFEIÇOAMENTO DE PESSOAL DE NÍVEL SUPERIOR, 2018).

As publicações para análise foram procuradas a partir do mecanismo de busca da Web of Science, utilizando-se como palavras-chaves, social learning (aprendizagem social) e (and) sustainable development (desenvolvimento sustentável) delimitando a busca para o período de 2009 a 2018 (10 anos). Após a pesquisa com as palavras-chave, foi utilizada a análise de resultados da Web of Science, com o intuito de obter as características gerais das publicações.

\subsection{MODELO CONCEITUAL}

O modelo conceitual do estudo está estabelecido nos principais elementos fornecidos pela base de dados Web of Science, os quais contemplam as características gerais das publicações e elementos de índices, h-b e m, que fazem parte da análise dos dados e dos hot topics que serão apresentados nas seções a seguir.

Quadro 2 - Modelo conceitual para análise bibliométrica

\begin{tabular}{|c|c|c|}
\hline \multicolumn{2}{|c|}{ Características gerais das publicações } & Número de citações de cada publicação \\
\hline Áreas temáticas & Instituições & Índice $\mathrm{h}-\mathrm{b}$ \\
\hline Tipos de documentos & Agências financiadoras & \\
\hline Ano das publicações & Países & \\
\hline Autores & Idiomas & \\
\hline Título das fontes & & \\
\hline
\end{tabular}

Fonte: Elaborado pelos autores

Para análise dos índices h-b e m, foram utilizadas as definições de Banks (2006). Segundo o autor, o índice $h$-b é obtido por meio do número de citações de um tópico ou combinação em certo período, listados em ordem decrescente de citações e, define-se o número de artigos com citações maiores ou iguais a esse número. Já o índice $m$ é obtido com a divisão do índice $h-b$ pelo período de anos que se deseja obter informações. O Quadro 3 evidencia estas definições.

Quadro 3 - Definições para classificação de hot topics

\begin{tabular}{|c|l|}
\hline Índice $\mathrm{m}$ & \multicolumn{1}{c|}{ Tópico/combinação } \\
\hline $0<\mathrm{m} \leq 0,5$ & $\begin{array}{l}\text { Pode ser de interesse para pesquisadores em um campo específico de pesquisa, o qual } \\
\text { engloba uma comunidade pequena; }\end{array}$ \\
\hline $0,5<\mathrm{m} \leq 2$ & $\begin{array}{l}\text { Provavelmente pode se tornar um "hot topic" como área de pesquisa, no qual a } \\
\text { comunidade é muito grande ou o tópico/combinação apresenta características muito } \\
\text { interessantes; }\end{array}$ \\
\hline $\mathrm{m} \geq 2$ & $\begin{array}{l}\text { É considerado um "hot topic", tópico exclusivo com alcance não apenas na sua própria área } \\
\text { de pesquisa e é provável que tenha efeitos de aplicação ou características únicas. }\end{array}$ \\
\hline
\end{tabular}

Fonte: Banks (2006).

Neste estudo, conforme as definições de Banks (2006) serão considerados hot topics as combinações com índice $m \geq 2$.

\section{ANÁLISE DOS RESULTADOS}

A partir das 2.024 publicações encontradas na pesquisa realizada, os procedimentos de análises e levantamento de indicadores bibliométricos, para maior entendimento do tema abordado no estudo, seguiram o conteúdo evidenciado no Quadro 2. Inicialmente, as principais áreas temáticas relacionadas ao tema da pesquisa podem ser visualizadas na Tabela 1.

RGO - Revista Gestão Organizacional, Chapecó, v. 13, n. 3, p. 184-203, set./dez. 2020. 
Tabela 1 - Principais áreas temáticas

\begin{tabular}{|c|c|c|}
\hline & Área temática & $\begin{array}{c}\text { No de } \\
\text { publicações }\end{array}$ \\
\hline 10 & Education educational research (Pesquisa educacional) & 498 \\
\hline 20 & Environmental sciences ecology (Ciências ambientais e ecologia) & 342 \\
\hline 30 & Environmental Studies (Estudos ambientais) & 285 \\
\hline $4 \stackrel{0}{0}$ & $\begin{array}{l}\text { Green Sustainable Science Technology (Tecnologia e ciências sustentáveis } \\
\text { verdes) }\end{array}$ & 280 \\
\hline 50 & Management (Gestão) & 152 \\
\hline 60 & Engeneering Environmental (Engenharia ambiental) & 108 \\
\hline $7 \underline{0}$ & Social Sciences Interdisciplinary (Interdisciplinaridade das ciências sociais) & 103 \\
\hline 80 & Business (Negócios) & 102 \\
\hline 9o & Regional Urban Planning (Planejamento urbano regional) & 100 \\
\hline 10 - & Education Scientific Disciplines (Disciplinas de educação científica) & 91 \\
\hline 11 운 & Ecology (Ecologia) & 87 \\
\hline 120 & Economics (Economia) & 85 \\
\hline 13 운 & Development Studies (Estudos em desenvolvimento) & 58 \\
\hline 140 & $\begin{array}{l}\text { Public Environmental Occupational Health (Saúde ocupacional e ambiental } \\
\text { pública) }\end{array}$ & 57 \\
\hline 150 & Water Resources (Recursos de água) & 51 \\
\hline 160 & Engineering Multidisciplinary (Engenharia multidisciplinar) & 50 \\
\hline 170 & Geography (Geografia) & 50 \\
\hline 180 & Urban Studies (Estudos urbanos) & 48 \\
\hline 190 & Energy Fuels (Combustíveis energéticos) & 45 \\
\hline 20 o & Agriculture Multidisciplinary (Agricultura multidisciplinar) & 40 \\
\hline
\end{tabular}

Fonte: Elaborado pelos autores.

Sendo a aprendizagem social uma abordagem educacional, a maior parte dos artigos encontra-se na área temática Education educational research (Pesquisa educacional), totalizando 498 artigos. Além disso, a segunda área temática que mais se destaca é a Environmental sciences ecology (Ciências ambientais e ecologia), com um total de 342 artigos, o que pode ser vinculado a forte associação de desenvolvimento sustentável com as dimensões ambiental e ecológica da sustentabilidade.

Ainda, as áreas temáticas Environmental Studies (Estudos ambientais), Green Sustainable Science Technology (Tecnologia e ciências sustentáveis verdes) e Management (Gestão) também apresentaram representatividade significativa, o que pode indicar o interesse de áreas mais aplicadas e técnicas no estudo da temática. Da mesma forma, a tendência de inserção do DS e aprendizagem social nas engenharias e ciências sociais pode ser percebida a partir do número de artigos relacionados às áreas de Engeneering Environmental (Engenharia ambiental) e Social Sciences Interdisciplinary (Interdisciplinaridade das ciências sociais), com 108 e 103 artigos respectivamente.

$\mathrm{Na}$ Tabela 2, são apresentados os principais tipos de documentos em que os artigos foram publicados. 
Aprendizagem social e desenvolvimento sustentável: um estudo bibliométrico no Web of Science da última década

Tabela 2 - Principais tipos de documentos

\begin{tabular}{|c|c|c|c|}
\hline & Tipos de publicação & Frequência & Percentual \\
\hline 10 & Article & 1346 & $66,50 \%$ \\
\hline 20 & Proceeding paper & 595 & $29,39 \%$ \\
\hline 3음 & Review & 91 & $4,50 \%$ \\
\hline 40 & Editorial material & 19 & $0,93 \%$ \\
\hline 50 & Book chapter & 4 & $0,20 \%$ \\
\hline 60 & Book review & 1 & $0,05 \%$ \\
\hline 70 & Letter & 1 & $0,05 \%$ \\
\hline 80 & Retracted publication & 1 & $0,05 \%$ \\
\hline
\end{tabular}

Fonte: Elaborado pelos autores.

De acordo com a Tabela 2, nota-se que a predominância de documentos se deu no formato de artigo, com uma representatividade de aproximadamente $66,5 \%$. Na sequência, têm-se os Proceedings Paper com 595 documentos e representatividade de aproximadamente 29,39\%. Já a Figura 1, apresenta a distribuição das publicações por ano.

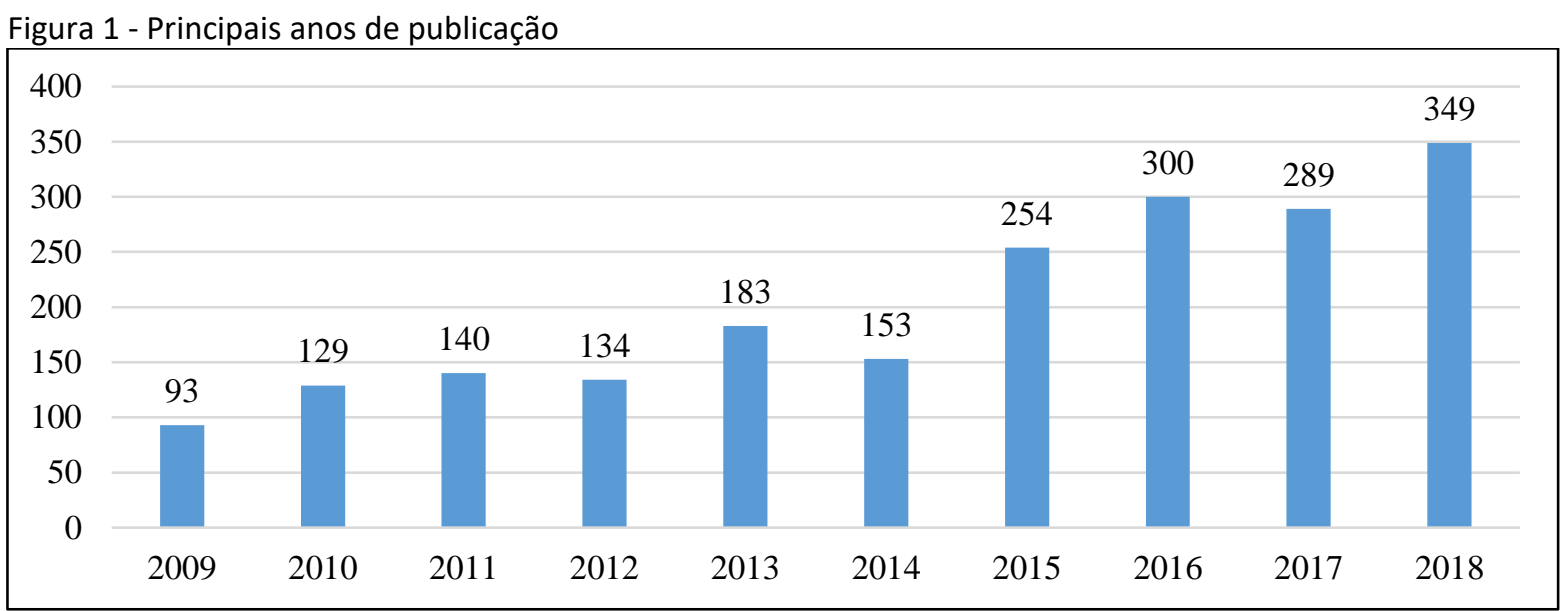

Fonte: Elaborado pelos autores.

Em relação ao ano das publicações, verifica-se uma tendência de crescimento no número de artigos publicados desde 2009, apresentando quedas apenas nos anos de 2012, 2014 e 2017. As publicações atingiram o auge no ano de 2018, apresentando um elevado aumento a partir do ano de 2015 . Os dados indicam que essa é uma temática de interesse recente, com publicações de maior impacto concentradas nos últimos 4 anos. Na sequência, são apresentados os principais autores que publicaram a respeito da temática pesquisada.

Tabela 3 - Principais autores

\begin{tabular}{|c|c|c|c|}
\hline Autores & Publicações & Autores & Publicações \\
\hline 1ㅇ Robert Axelsson & 11 & 11ㅇ Ignacio Carmenado De Rios & 4 \\
\hline 2o Marine Elbakidze & 11 & 12ㅇ Joaquin Gutierrez-Yurrita & 4 \\
\hline 3o Per Angelstam & 10 & 13ㅇ Ralph Hansmann & 4 \\
\hline 4ㅇ Jana Dlouha & 8 & 14으 M Jones & 4 \\
\hline 5o Kjell Andersson & 6 & 15은ouis Lebel & 4 \\
\hline 60 Douglas Bardsley & 6 & 160 Cees Leeuwis & 4 \\
\hline 7ㅇ Jiri Dlouhy & 6 & 17음odrigo Lozano & 4 \\
\hline 80 Matthias Barth & 5 & 18음orn Nykvist & 4 \\
\hline 9o Ioan Fazey & 5 & 19 Claudia Pahl Hostl & 4 \\
\hline 10ㅇ Pieter Beers & 4 & 20ㅇ Ryan Plummer & 4 \\
\hline
\end{tabular}

Fonte: Elaborado pelos autores.

RGO - Revista Gestão Organizacional, Chapecó, v. 13, n. 3, p. 184-203, set./dez. 2020. 
Conforme pode-se observar na Tabela 3, não há apenas um autor que se destaque em número de publicações sobre a temática abordada. Os três autores mais produtivos são Robert Axelsson (Suécia), Marine Elbakidze (Suécia) e Per Angelstam (Suécia). Nota-se, ainda que os três autores de destaque são da Suécia e que os autores Marine Elbakidze e Per Angelstam são da instituição Swedish University of Agricultural Sciences, que se sobressai entre as instituições que mais publicam sobre a temática, conforme Tabela 4.

Tabela 4 - Principais Instituições

\begin{tabular}{|c|c|c|c|}
\hline Instituição & $\begin{array}{c}\text { № de } \\
\text { Publicações }\end{array}$ & Instituição & $\begin{array}{c}\text { No de } \\
\text { Publicações } \\
\end{array}$ \\
\hline 1ㅇ Wageningen University & 48 & 14을 University of British Columbia & 14 \\
\hline 2ㅇ University of London & 29 & 150 University College London & 13 \\
\hline 3o University of Stockholm & 26 & 16ㅇ University of North Carolina & 13 \\
\hline 4ㅇ Arizona State University & 20 & 170 Utrecht University & 13 \\
\hline $\begin{array}{l}\text { 5o Swedish University of } \\
\text { Agricultural Sciences }\end{array}$ & 19 & 18ㅇ Monash University & 12 \\
\hline $\begin{array}{l}\text { 60 Commonwealth Scientific } \\
\text { Industrial Research Organisation }\end{array}$ & 18 & $\begin{array}{l}\text { 19o Pennsylvania Commonwealth System } \\
\text { of Higher Education }\end{array}$ & 12 \\
\hline 7ㅇ Leuphana University Luneburg & 18 & 20 - State University System of Florida & 12 \\
\hline $\begin{array}{l}\text { 8o Polytechnic University of } \\
\text { Madrid }\end{array}$ & 16 & 21ㅇ University of Manchester & 12 \\
\hline 9o University of Basque Country & 16 & 220 University of Melbourne & 12 \\
\hline 10 Helmholtz Association & 15 & 23ㅇA Aalborg University & 11 \\
\hline 11은 University of Adelaide & 15 & 24을 Australian National University & 11 \\
\hline $\begin{array}{l}\text { 12ㅇ University of California } \\
\text { System }\end{array}$ & 15 & $\begin{array}{l}\text { 25o Bucharest Academy of Economic } \\
\text { Studies }\end{array}$ & 11 \\
\hline 13은 University of Leeds & 15 & & \\
\hline
\end{tabular}

Fonte: Elaborado pelos autores.

Além da Swedish University of Agricultural Sciences (localizada na Suécia), outras instituições se destacam na publicação de trabalhos sobre a temática, são elas Wageningen University (Holandesa), University of London (Inglesa) e University of Stockholm (Suéca). No que diz respeito as principais fontes de publicação acerca do tema, a Tabela 05 evidencia os resultados. É importante salientar que a temática estudada encontra os principais estudos sendo realizados no Norte da Europa, seguido de universidades Americanas e Australianas. Estes resultados demonstram, em especial, as escolas que possuem na filosofia práticas para o desenvolvimento sustentável e iniciativas que geram aprendizagem em diferentes níveis de conhecimento nas mais diversas áreas.

De acordo com a Tabelas 5, no que tange às principais fontes de publicação, pode-se averiguar que o Journal de maior destaque em número de publicações sobre a temática é o Journal of Cleaner Production, uma revista internacional, transdisciplinar, voltada para a pesquisa e a prática da Produção Mais Limpa, Ambiental e Sustentável (ELSEVIER, 2018), que apresenta 78 publicações sobre o tema. Outro periódico que se destaca é a Sustainability, com 67 trabalhos. Além disso, pode-se notar que temática aparece significativamente em publicações em eventos, a saber: 49 publicações no Inted proceedings, conferência Internacional de Tecnologia, Educação e Desenvolvimento (INTED, 2018), e 44 estudos na Edulearn proceedings que consiste em uma das maiores conferências internacionais de educação para professores, pesquisadores, tecnólogos e profissionais do setor educacional (EDULEARN, 2018). Os journals que lideram a temática, também lideram outras áreas de 
Aprendizagem social e desenvolvimento sustentável: um estudo bibliométrico no Web of Science da última década

pesquisas, como sustentabilidade, inovação, universidades sustentáveis, empresas sustentáveis, etc. Salienta-se que a temática do desenvolvimento sustentável em aprendizagem social são áreas que possuem conexão na literatura, ações práticas e iniciativas fundamentais para os avanços das organizações.

Tabela 5 - Principais Fontes de Publicação

\begin{tabular}{|c|c|c|c|}
\hline Título da Fonte & $\begin{array}{c}\text { № de } \\
\text { Publicações }\end{array}$ & Título da Fonte & $\begin{array}{c}\text { NNo de } \\
\text { Publicações }\end{array}$ \\
\hline 10 Journal of Cleaner Production & 78 & $\begin{array}{l}14 \text { o Inted } 2017 \quad 11 \text { th International } \\
\text { Technology Education and Development } \\
\text { Conference }\end{array}$ & 11 \\
\hline 20 Sustainability & 67 & 15으 Sustainability Science & 11 \\
\hline 3o Inted proceedings & 49 & $\begin{array}{l}\text { 16- Edulearn } 16 \text { 8th International } \\
\text { Conference on Education and New } \\
\text { Learning Technologie }\end{array}$ & 10 \\
\hline 40 Edulearn Proceedings & 44 & $\begin{array}{l}\text { 170 International Journal of Agricultural } \\
\text { Sustainability }\end{array}$ & 10 \\
\hline 5o Ecology and Society & 37 & 18ㅇ Land Use Policy & 10 \\
\hline $\begin{array}{lll}60 \quad \text { International journal of } \\
\text { sustainability in higher education }\end{array}$ & 34 & $\begin{array}{l}\text { 19- Advances in Social Science Education } \\
\text { and Humanities Research }\end{array}$ & 9 \\
\hline $\begin{array}{l}\text { 70 Procedia Social and Behavioral } \\
\text { Sciences }\end{array}$ & 30 & $\begin{array}{l}20 \text { - Iceri2016 9th International } \\
\text { Conference of Education Research and } \\
\text { Innovation }\end{array}$ & 9 \\
\hline 8 Iceri Proceedings & 25 & $\begin{array}{l}210 \quad \text { Journal of Environmental } \\
\text { Management }\end{array}$ & 9 \\
\hline 9o Asee Annual Conference Exposition & 14 & $\begin{array}{l}\text { 22ㅇ Journal of Environmental Planning } \\
\text { and Management }\end{array}$ & 9 \\
\hline 10 Environmental Education Research & 14 & 23응 Ocean Coastal Management & 9 \\
\hline $\begin{array}{l}11 \text { o Inted } 2016 \text { 10th international } \\
\text { technology education and } \\
\text { development conference }\end{array}$ & 12 & $\begin{array}{l}\text { 240 10th International Conference of } \\
\text { Education Research and Innovation } \\
\text { Iceri2017 }\end{array}$ & 8 \\
\hline 12 - International Review of Education & 12 & $\begin{array}{l}\text { 250 12th International Technology } \\
\text { Education and Development Conference }\end{array}$ & 8 \\
\hline 13을 Environmental Science Policy & 11 & & \\
\hline
\end{tabular}

Fonte: Elaborado pelos autores.

A próxima característica a ser analisada é a agência de financiamento que apresentou maior número de trabalhos. Os resultados encontrados podem ser visualizados na Tabela 6.

De acordo com as informações expostas na Tabela 6, a agência que apresentou um maior número de trabalhos sobre aprendizagem social e desenvolvimento sustentável foi a National Natural Science Foundation of China, localizada na China com 17 ocorrências. Em segundo lugar tem-se a National Science Foundation, dos EUA, com 14 trabalhos. Como terceira agência destaque evidencia-se a Economic and Social Research Council, do Reino Unido, com 13 publicações. As organizações de incentivos e investimentos em pesquisas Asiáticas estão nos últimos anos liderando importantes resultados na ciência, seguidas de organizações Europeias e Americanas. Destaca-se que as políticas governamentais são muito importantes neste processo, além de doações e incentivos empresariais para promover projetos e Pesquisas \& Desenvolvimento. Na América do Sul, as iniciativas são desenvolvidas por órgãos governamentais como Capes, Cnpq e agências estaduais, porém com recursos limitados e menor escala de competividade em relação às primeiras colocadas. 
Tabela 6 - Principais agências de financiamento

\begin{tabular}{|c|c|c|c|}
\hline Agências de financiamento & Registros & Agências de financiamento & Registros \\
\hline $\begin{array}{l}\text { 10 National Natural Science Foundation of } \\
\text { China }\end{array}$ & 17 & 140 Swedish Institute & 5 \\
\hline 2o National Science Foundation & 14 & 15 Medical Research Council & 4 \\
\hline 3o Economic and Social Research Council & 13 & 160 Swedish Research Council & 4 \\
\hline $\begin{array}{l}\text { 40 Engineering and Physical Sciences Research } \\
\text { Council }\end{array}$ & 11 & $\begin{array}{l}\text { 17o Swedish Research Council for } \\
\text { Environment Agricultural Sciences } \\
\text { and Spatial Planning FORMAS }\end{array}$ & 4 \\
\hline 5o European Commission & 11 & 18ㅇ Academy of Finland & 3 \\
\hline 6o European Union & 8 & 19o Basque Government & 3 \\
\hline 70 Swedish Research Council Formas & 8 & $\begin{array}{l}20 \text { Economic and Social } \\
\text { Research Council ESRC }\end{array}$ & 3 \\
\hline 8 o Formas & 6 & 21을 European Social Fund & 3 \\
\hline 9o Mistra & 6 & 22ㅇ Futura Foundation & 3 \\
\hline 100 Australian Research Council & 5 & $\begin{array}{l}\text { 23o German Academic Exchange } \\
\text { Service DAAD }\end{array}$ & 3 \\
\hline 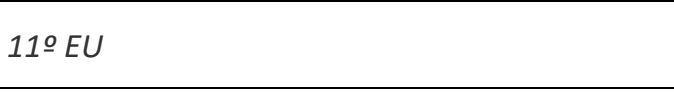 & 5 & $\begin{array}{l}\text { 240 Italian Ministry of Agricultural } \\
\text { Food and Forestry Policies }\end{array}$ & 3 \\
\hline $12^{\circ}$ Economic and Social Research Council Esrc & 5 & 250 Ministry of Education Malaysia & 3 \\
\hline $\begin{array}{l}\text { 13o Social Sciences and Humanities Research } \\
\text { Council of Canada }\end{array}$ & 5 & & \\
\hline
\end{tabular}

Fonte: Elaborado pelos autores.

$\mathrm{Na}$ Tabela 7 são apresentados os resultados referentes aos países que possuem publicações relacionadas ao tema pesquisado.

Tabela 7 - Principais países

\begin{tabular}{|c|c|c|c|}
\hline Países & № de Artigos & Países & № de Artigos \\
\hline 10 Estados Unidos da América & 344 & 14으frica do Sul & 55 \\
\hline 20 Inglaterra & 226 & 15오 Suíça & 50 \\
\hline 3o Austrália & 171 & 160 Índia & 45 \\
\hline 4o China & 147 & 17으úustria & 42 \\
\hline 5o Alemanha & 133 & 18요 Indonésia & 39 \\
\hline 6o Espanha & 130 & 19o Bélgica & 38 \\
\hline 70 Holanda & 127 & 20 Portugal & 38 \\
\hline 8ㅇ Canada & 114 & 21을éxico & 36 \\
\hline 9ㅇ Suécia & 106 & 22ㅇ Finlândia & 33 \\
\hline 10ㅇ Itália & 79 & 23oMalásia & 33 \\
\hline 110 Brasil & 72 & 240 Tailândia & 32 \\
\hline 12 França & 62 & 25№ Noruega & 31 \\
\hline 13으 Romênia & 59 & & \\
\hline
\end{tabular}

Fonte: Elaborado pelos autores.

No tocante ao número de publicações por países, os Estados Unidos da América lideram o ranking de publicações, com 344 trabalhos, seguidos da Inglaterra, Austrália, China e Alemanha. Destaca-se, ainda, neste cenário a diversidade de países e suas localizações. Além disso, ressalta-se que o Brasil está presente entre os 25 principais países que pesquisam o tema, o que representa um aspecto positivo, haja vista que 72 publicações são provenientes do país. Os resultados no que tange aos países com maior número de publicações, são reflexos das agências de fomentos, investimentos em pesquisas, assim como tempo de atuação em pesquisas voltados para a temática. É importante salientar que as Universidades Americanas 
Aprendizagem social e desenvolvimento sustentável: um estudo bibliométrico no Web of Science da última década

e Europeias possuem em média 200 a 300 anos de atuação, enquanto as Universidades da América Latina, como as do Brasil são jovens, sendo a maioria com menos de 50 anos de existência. Os resultados aqui não visam realizar comparativos, mas é importante destacar que as iniciativas de pesquisas, incentivos e projetos com maior suporte permitem melhores resultados.

A Tabela 8 evidencia os principais idiomas em que os trabalhos são publicados.

Tabela 8 - Principais Idiomas

\begin{tabular}{|c|c|c|c|}
\hline Idioma & № Publicações & Idioma & № Publicações \\
\hline 1으 English & 1931 & 9o Latvian & 3 \\
\hline 2o Spanish & 41 & 10 Slovak & 3 \\
\hline 3ㅇ Portuguese & 10 & 11 Czech & 2 \\
\hline 4으 Russian & 7 & 12 French & 2 \\
\hline 5o Chinese & 5 & 13으ungarian & 2 \\
\hline 6ㅇ Croatian & 5 & 14음 Dutch & 1 \\
\hline 7ㅇ German & 5 & 15을 Estonian & 1 \\
\hline 8o Turkish & 4 & 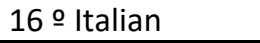 & 1 \\
\hline
\end{tabular}

Fonte: Elaborado pelos autores.

A partir dos dados evidenciados pode-se verificar que há uma grande predominância da língua inglesa (English), 1931 trabalhos. Além disso, 41 trabalhos sobre a temática foram publicados em espanhol (Spanish). A língua Portuguesa (Portuguese) está no terceiro lugar, com 10 publicações. As demais línguas, que aparecem com menor representatividade, não possuem diferenças significativas de representatividade. Por fim, para análise dos hot topics, investigou-se as publicações referentes a social learning (aprendizagem social) e sustainable development (desenvolvimento sustentável) na base de dados Web of Science e os principais tópicos de estudo relacionados a esta temática. A partir de uma análise prévia das publicações encontradas na Web of Science, foram selecionados 20 tópicos relacionados à temática.

Os tópicos identificados foram: Management (Gestão), Development Studies (Estudos de desenvolvimento), Environmental studies (Estudos ambientais), Environmental sciences (Ciências ambientais), Business (Negócios), Water resources (Recursos naturais), Urban studies (Estudos urbanos), Ecology (Ecologia), Education educational research (Pesquisa na educação), Social sciences interdisciplinary (Ciências sociais interdisciplinares), Economics (Economia), Engineering environmental (Engenharia ambiental), Regional Urban Planning (Planejamento urbano regional), Geography (Geografia), Engineering multidisciplinary (Engenharia multidisciplinar), Energy fuels (Combustíveis energéticos), Education scientific disciplines (Disciplinas científicas de educação), Green Sustainable Science Technology (Tecnologia e ciências sustentáveis verdes), Agriculture multidisciplinary (Agricultura multidisciplinar), Public environmental occupational health (Saúde ocupacional ambiental pública).

Posteriormente, foi realizada a combinação de cada tópico listado com os termos social learning e sustainable development, sendo calculado o total de publicações para cada combinação (tópico relacionado), o h-index e o coeficiente " $m$ ", conforme Tabela 9.

Conforme os cálculos dos índices h-b e m é possível mensurar o desempenho dos tópicos/combinações pesquisados tendo por base o número de citações que estas obtiveram (KELLY e JENNIONS, 2006). Baseando-se nas orientações de Banks (2006) é possível classificar como "hot topics" ou tópicos quentes as combinações Management (Gestão), Development Studies (Estudos de desenvolvimento), Environmental studies (Estudos ambientais), 
Environmental sciences (Ciências ambientais). As demais combinações, por apresentarem 0,5 $<\mathrm{m} \leq 2$, podem ser consideradas como "hot topics" emergentes, como áreas de pesquisa.

Tabela 9 - "Hot Topics" com Índice h-b e Índice m

\begin{tabular}{|c|c|c|c|}
\hline Área Temática & № Publicações & Índice h-b & Índice m \\
\hline 1ㅇManagement (Gestão) & 152 & 39 & 3,9 \\
\hline 2o Development Studies (Estudos de desenvolvimento) & 58 & 39 & 3,9 \\
\hline 3o Environmental studies (Estudos ambientais) & 285 & 28 & 2,8 \\
\hline 4o Environmental sciences (Ciências ambientais) & 342 & 24 & 2,4 \\
\hline 5o Business (Negócios) & 102 & 19 & 1,9 \\
\hline 60 Water resources (Recursos naturais) & 51 & 19 & 1,9 \\
\hline 7o Urban studies (Estudos urbanos) & 48 & 14 & 1,4 \\
\hline 8o Ecology (Ecologia) & 87 & 13 & 1,3 \\
\hline 9o Education educational research (Pesquisa na educação) & 498 & 11 & 1,1 \\
\hline $\begin{array}{l}\text { 10o Social sciences interdisciplinary (Ciências sociais } \\
\text { interdisciplinares) }\end{array}$ & 103 & 10 & 1,0 \\
\hline $11^{\circ}$ Economics (Economia) & 85 & 10 & 1,0 \\
\hline 12을 Engineering environmental (Engenharia ambiental) & 108 & 9 & 0,9 \\
\hline 13ㅇ Regional Urban Planning (Planejamento urbano regional) & 100 & 6 & 0,6 \\
\hline 14ㅇGeography (Geografia) & 50 & 6 & 0,6 \\
\hline 15을 Engineering multidisciplinary (Engenharia multidisciplinar) & 50 & 4 & 0,4 \\
\hline 16을ergy fuels (Combustíveis energéticos) & 45 & 4 & 0,4 \\
\hline $\begin{array}{l}\text { 17o Education scientific disciplines (Disciplinas científicas de } \\
\text { educação) }\end{array}$ & 91 & 3 & 0,3 \\
\hline $\begin{array}{l}\text { 180 Green Sustainable Science Technology (Tecnologia e ciências } \\
\text { sustentáveis verdes) }\end{array}$ & 280 & 2 & 0,2 \\
\hline 19ㅇ Agriculture multidisciplinary (Agricultura multidisciplinar) & 40 & 1 & 0,1 \\
\hline $\begin{array}{l}\text { 20o Public environmental occupational health (Saúde ocupacional } \\
\text { ambiental pública) }\end{array}$ & 57 & 0 & 0,0 \\
\hline
\end{tabular}

Fonte: Elaborado pelos autores.

Os resultados apresentados na análise de hot topics são importantes para o progresso dos estudos e avaliar o quanto a ciência no que tange aos aspectos do desenvolvimento sustentável evoluíram nos últimos anos. A área de gestão que obteve melhores resultados é um dos principais campos de estudo que contemplam aspectos como: gestão de energia, gestão das mudanças climáticas, gestão da inovação e da sustentabilidade, gestão comportamental e sustentabilidade, gestão financeira e social, governança e sustentabilidade, etc. Todas essas áreas possuem aspectos ligados à aprendizagem social, pois estas áreas de estudo buscam promover iniciativas, desenvolver práticas e criar mudanças de comportamento. As outras áreas que também obtiveram bons resultados foram os estudos de desenvolvimento, estudos ambientais e ciências ambientais. Como pode-se observar, a área ambiental é a base dos estudos para o desenvolvimento sustentável e, assim como a área de gestão, ambas têm sido promotoras não somente da ciência, mas de ações práticas empresariais que permitem aprendizagem organizacional, seja no viés técnico, assim como, comportamental em mudanças na redução de desperdícios, técnicas para reaproveitamento de matérias, entre outros aspectos.

\section{CONSIDERAÇÕES FINAIS}

O presente estudo teve por objetivo analisar as características das publicações sobre social learning (aprendizagem social) e sustainable development (desenvolvimento 
Aprendizagem social e desenvolvimento sustentável: um estudo bibliométrico no Web of Science da última década

sustentável) na base de dados Web of Science, no período de 2009 a 2018 e, ainda, identificar quais os tópicos mais relevantes relacionados à aprendizagem social e ao desenvolvimento sustentável que estão sendo investigados (hot topics). Neste sentido, foi desenvolvido um estudo bibliométrico, por meio da base de dados Web of Science, relacionando os tópicos citados, identificando 2024 publicações, em sua maioria composta por artigos, que representam $66,50 \%$ do total encontrado.

Entre os principais resultados pode-se destacar o crescimento da produção associada ao tema nos últimos anos, com uma pequena queda no ano de 2017 em relação a 2016. Os periódicos Journal of cleaner production, Sustainability, e os anais do Edulearn proceedings e Inted proceedings se destacam entre aqueles que possuem o maior número de publicações. Com relação às áreas temáticas, a pesquisa educacional (Education educational Research) e Ciências ambientais e ecologia (Environmental sciences ecology) se relevaram com maior número de publicações relacionadas aos temas pesquisados.

Os Estados Unidos da América lideram o ranking de países que publicam os tópicos da aprendizagem social e do desenvolvimento sustentável. No entanto a Inglaterra, Austrália, China e Alemanha também se destacam nas pesquisas. Dentre as três instituições que mais publicam a respeito dos temas, Wageningen University, University of Stockholm e University of London, apenas a última pertence ao país que aparece em primeiro lugar (EUA). Os três autores que mais publicam sobre os temas relacionados são Robert Axelsson, Marine Elbakidze e Per Angelstam. Torna-se interessante ressaltar que os três autores possuem vínculo com universidades suecas.

Com relação aos hot topics, foram identificadas quatro áreas temáticas, que juntamente com a aprendizagem social e o desenvolvimento sustentável, podem ser considerados tópicos exclusivos, com alcance em diversas áreas do conhecimento ou características únicas: Management (Gestão), Development Studies (Estudos de desenvolvimento), Environmental studies (Estudos ambientais) Environmental sciences (Ciências ambientais).

A aprendizagem social e o desenvolvimento sustentável, são dois temas que em tempos recentes estão em voga, em diferentes contextos e áreas de estudos. No contexto da gestão de organizações, que contempla áreas técnicas, estratégicas e comportamentais, muitos gestores estão buscando maior empregabilidade de ações estratégicas voltadas para melhor aproveitamento e redução do comprometimento dos recursos ambientais. Compreender como as pessoas aprendem individualmente ou em grupo, poderá trazer inúmeros benefícios, em especial nos aspectos de liderança, motivação e qualidade de vida para os colaboradores envolvidos.

Com os dados obtidos pelo estudo, torna-se possível identificar a utilização da aprendizagem social para o desenvolvimento sustentável, na gestão, em aspectos como gestão de energia, gestão das mudanças climáticas, gestão da inovação e da sustentabilidade, gestão comportamental e sustentabilidade, gestão financeira e social, governança e sustentabilidade, entre outras. Como pode-se observar, a área ambiental é a base dos estudos para a temática do desenvolvimento sustentável e ambas têm sido promotoras não somente da ciência, mas de ações práticas empresariais que permitem também aprendizagem organizacional, seja no viés técnico, assim como, comportamental em mudanças na redução de desperdícios e técnicas para reaproveitamento de materiais.

O presente estudo se diferencia ao realizar a análise das características gerais das publicações que envolvem os termos "aprendizagem social" e "desenvolvimento sustentável", no período entre 2009 e 2018, uma vez que não foram encontradas investigações semelhantes na literatura. 
Como limitação deste estudo, tem se a utilização de apenas uma base de dados. Desta forma, sugere-se a utilização de um maior número de recursos, como por exemplo, eventos nacionais e internacionais, bem como outras bases de dados. Para pesquisas futuras, sugerese estudar a temática em outras bases, visando verificar se há outros estudos neste enfoque. Também é possível estudar as relações entre os aspectos teóricos, métodos utilizados, abordagem teórica, entre outros detalhes.

\section{REFERÊNCIAS}

ALVARADO. R. U. A Lei de Lotka na bibliometria brasileira. Ciência da Informação, v. 31, n. 2, 2002.

AMARAL, D. G.; BRUNSTEIN, J. Aprendizagem social para sustentabilidade: a experiência de um programa empresarial de mulheres empreendedoras em situação de pobreza. Revista de Gestão Social e Ambiental, v. 11, n. 3, p. 2-20, 2017. DOI:

https://doi.org/10.24857/rgsa.v11i3.1339.

AVILA, L, V., FACCO, A, L., BENTO, M, A., ARIGONY, M, M., OBREGON, S, L., TREVISAN, M. Sustainability and education for sustainability: an analysis of publications from the last decade. Environmental Quality Management, v. 17, n. 3, p. 107-118, 2018. DOI: https://doi.org/10.1002/tqem.21537

BANDURA, A. Social learning theory. Englewood Cliffs, NJ: Prentice-Hall, 1977.

BANKS, M. G. An extension of the Hirsch index: Indexing scientific topics and compounds. Scientometrics, v. 69, n. 1, p. 161-168, 2006. DOI: https://doi.org/10.1007/s11192-0060146-5

BEBBINGTON, J.; UNERMAN, J. Achieving the United Nations Sustainable Development Goals: An enabling role for accounting research. Accounting, Auditing \& Accountability Journal, v. 31, n. 1, p. 2-24, 2017. DOI: https://doi.org/10.1108/AAAJ-05-2017-2929

CAPRA, F. Foreword. In: WALS, A. E. J. Social learning: towards a sustainable world. Wageningen: Wageningen Academic Publishers, 2007.

CARS, M.; WEST, E. E. Education for sustainable society: attainments and good practices in Sweden during the United Nations decade for education for sustainable development. Environment, Development and Sustainability, v. 17, p. 1-21, 2014. DOI: https://doi.org/10.1007/s10668-014-9537-6

CASTRO, C. J. Sustainable development: mainstream and critical perspectives. Organization \& Environment, v. 17, n. 2, p. 195-225, jun. 2004. DOI: https://doi.org/10.1177/1086026604264910

CASTRO FILHO, C. M. de. Agenda 2030 para o desenvolvimento sustentável: uma leitura de política pública na clave da biblioteca escolar. Revista Digital Biblioteconomia e Ciência da Informação, v. 16, n. 3, p. 355-372, 2018. DOI: https://doi.org/10.20396/rdbci.v16i3.8650931 
Aprendizagem social e desenvolvimento sustentável: um estudo bibliométrico no Web of Science da última década

CAVAGNARO, E.; CURIEL, G. The Three Levels of Sustainability. Sheffield: Greenleaf Publishing, 2012

CMMAD - Comissão Mundial Sobre o Meio Ambiente e Desenvolvimento. Relatório Nosso Futuro Comum. 2. ed. Rio de Janeiro: FGV, 1991. 226 p.

COORDENAÇÃO DE APERFEIÇOAMENTO DE PESSOAL DE NÍVEL SUPERIOR, 2018, Acervo. Disponível em:

https://www.periodicos.capes.gov.br/?option=com_pcollection \&mn=70\&smn=79\&cid=81. Acesso em: 19 mai. 2018.

D’ANGELO, M, J.; BRUNSTEIN, J. Social learning for sustainability: supporting sustainable business in Brazil regarding multiple social actors, relationships and interests. International Journal of Sustainable Development \&World Ecology, v. 21, n. 3, p. 273-289, 2014. DOI: https://doi.org/10.1080/13504509.2014.902868

DIDHAM, R. J.; OFEI-MANU, P.; NAGAREO, M. Social learning as a key factor in sustainability transitions: The case of Okayama City. International Review of Education, v. 63, p 829-846, 2017.

EDULEARN. About Edulearn 2018. Disponível em: https://iated.org/edulearn/. Acesso em: 15 mai. 2018.

ELKINGTON, J. Cannibals with forks: the triple botton line of 21st century business. Oxford: Capstone: 1999.

ELSEVIER. Journal of Cleaner Production. Disponível em: https://www.journals.elsevier.com/journal-of-cleaner-production. Acesso em: maio de 2018.

FAVORETO, R. L.; AMÂNCIO-VIEIRA, S. F.; SHIMADA, A. P. A produção intelectual em RBV: Uma incursão bibliométrica nos principais periódicos internacionais. Revista Brasileira de Estratégia, v. 7, n. 1, p. 37-55, 2014.

FOLADORI, G. Limites do Desenvolvimento Sustentável. Campinas: Editora da UNICAMP, 2001.

GLASSER, $\mathrm{H}$. Minding the gap: the role of social learning in linking our stated desire for a more sustainable world to our everyday actions and policies. In: WALS A. E. J (editor). Social learning: towards a sustainable world. Wageningen: Wageningen Academic, 2007. 537 p.

GODOI, C. K.; FREITAS, S. F. A aprendizagem organizacional sob a perspectiva sócio-cognitiva: contribuições de Lewin, Bandura e Giddens. Revista de Negócios, v. 13, n. 4, p. 40-55, 2008. DOI: http://dx.doi.org/10.7867/1980-4431.2008v13n4p40-55 
GRIGGS, D.; SMITH, M. S.; ROCKSTRÖM, J.; ÖHMAN, M. C.; GAFFNEY, O.; GLASER, G.; KANIE, N.; STEFFEN, W.; SHYAMSUNDAR, P. An integrated framework for sustainable development goals. Ecology and Society, v. 19, n. 4, 2014. DOI: http://dx.doi.org/10.5751/ES-07082190449

HAIR J. R., J. F.; BABIN, B.; MONEY, A. H.; SAMOUEL, P. Fundamentos de métodos de pesquisa em administração. Porto Alegre: Bookman, 2005.

HARMONISING COLLABORATIVE PLANNING (HARMONICOP). Learning together to manage together: improving participation in water management, Osnabrück, 2005. Disponível em: https://www.ecologic.eu/sites/files/publication/2014/kranz_06_harmonicophandbook_en.p df. Acesso em: 20 abr. 2018.

HOPWOOD, B.; MELLOR, M.; O'BRIEN, G. Sustainable development: Mapping different approaches. Sustainable Development, v. 13, p. 38-52, 2005. DOI:

https://doi.org/10.1002/sd.244

INTED. International Technology, Education and Development Conference. Publications. Disponível em: https://iated.org/inted/publications. Acesso em: 18 mai. 2018.

JACOBI, P. R. Aprendizagem social, desenvolvimento de plataformas de múltiplos atores e governança da água no brasil. Revista Internacional Interdisciplinar INTERthesis, v. 7, n. 1, p. 69-95, 2010. DOI: https://doi.org/10.5007/1807-1384.2010v7n1p69

KEEN, M.; BROWN, V; DYBALL, R. Social learning in environmental management: towards a sustainable future. Londres: Earthscan, 2005.

KELLY, C. D.; JENNIONS, M. D. The $h$ index and career assessment by numbers. Trends in Ecology \& Evolution, v. 21, n. 4, p. 167-170, 2006.

KILVINGTON, M. Social learning as framework for building capacity to work on complex environmental management problems. Landcare Research, Nova Zelândia, 2007. Disponível em: http://www.landcareresearch.co.nz/publications/researchpubs. Acesso em: 20 jan. 2018.

KILVINGTON, M. Building Capacity for Social Learning in Environmental Management. Orientadores: Roy Montgomery e Stefanie Rixecker. 2010. 365f. Tese (Doutorado em Filosofia) - Lincoln University, Canterbury, Nova Zelândia, 2010. Versão eletrônica.

MACEDO, M. A. S.; CASA NOVA, S. P.; ALMEIDA, K. Mapeamento e análise bibliométrica da utilização da análise envoltória de dados (DEA) em estudos das áreas de contabilidade e administração. Contabilidade, Gestão e Governança, v. 12, n. 3, p. 87-101, 2009.

MALHOTRA, N. Pesquisa de marketing: uma orientação aplicada. 4. ed. Porto Alegre: Bookman, 2006. 
Aprendizagem social e desenvolvimento sustentável: um estudo bibliométrico no Web of Science da última década

MORETTI, S. L. A.; CAMPANÁRIO, M. A. A produção intelectual brasileira em responsabilidade social empresarial - RSE sob a ótica da bibliometria. Revista de Administração Contemporânea, v. 13, n. 8, p. 68-86, 2009. DOI: https://doi.org/10.1590/S1415-65552009000500006

ONU. Transformando Nosso Mundo: A Agenda 2030 para o Desenvolvimento Sustentável, 2015. Disponível em: http://www.agenda2030.com.br/biblioteca/Agenda2030-completosite.pdf >. Acesso em: 24 fev. 2017.

REED, M. K.; EVELY, A. C.; CUNDILL, G.; FAZEY, I.; GLASS, J.; LAING, A.; NEWIG, J.; PARRISH, B.; PRELL, C.; RAYMOND, C.; STRINGER, L. C. What is Social Learning? Ecology and Society, v. 12, n. 2, 2010. DOI: https://doi.org/10.5751/ES-03564-1504r01

RODELA, R.; CUNDILL, G.; WALS, A. E. J. An analysis of the methodological underpinnings of social learning research in natural resource management. Ecological Economics, v. 77, p 1626, 2012.

ROMEIRO, A. R. Desenvolvimento sustentável: uma perspectiva econômico-ecológica. Estudos Avançados, v. 26, n. 74, p. 65-92, 2012.

ROSTAING, H. La bibliométrie et sés techniques. Toulouse: Sciences de la Société, 1997.

SACHS, I. Desenvolvimento: includente, sustentável e sustentado. Rio de Janeiro: Garamond, 2004.

SEGHEZZO, L. The five dimensions of sustainability. Environmental Politics, v. 18, n. 4, p. 539-556, 2009. DOI: https://doi.org/10.1080/09644010903063669

SILVA, M. R. (2004). Análise bibliométrica da produção científica docente do programa de pós-graduação em educação especial/UFSCAR: 1998-2003. Orientador: Maria Cristina Hayashi. 2004. Dissertação (Mestrado em Educação Especial) - Universidade Federal de São Carlos, 2004.

WALS, A. E. J.; HOEVEN, N. V. D.; BLANKEN, H. The acoustics of social learning: designing learning processes that contribute to a more sustainable world. Wageningen: Wageningen Academic Publishers, 2009.

WALS, A. E. J.; VAN DER LEIJ, T. Introduction. In: WALS, A. E. J. Social learning: towards a sustainable world. Wageningen: Wageningen Academic Publishers, 2007.

WENGER, E.; TRAYNER, B. Comunities of practice a brief introduction. 2015. Disponível em: http://wenger-trayner.com/introduction-to-communities-of-practice/. Acesso em: 20 out. 2018.

XAVIER, T. R.; WITTMANN, M. L.; INÁCIO, R. O.; KERN, J. Desenvolvimento regional: uma análise sobre a estrutura de um consórcio intermunicipal. Revista de Administração Pública, v. 47 n. 4, p. 1-14, 2013. DOI: https://doi.org/10.1590/S0034-76122013000400011 